\title{
Patterns of Care in Adjuvant Therapy for Resected Oral Cavity Squamous Cell Cancer in Elderly Patients
}

\author{
Erqi L. Pollom, MD, MS $\#^{\star}$, Alexander L. Chin, MD, MBA ${ }^{\#^{*}}$, Nancy Y. Lee, MD $^{\dagger}$, and C. Jillian \\ Tsai, MD, $\mathbf{P h D}^{\dagger}$ \\ *Department of Radiation Oncology, Stanford University School of Medicine, Stanford, California \\ †Department of Radiation Oncology, Memorial Sloan Kettering Cancer Center, New York, New \\ York \\ \# These authors contributed equally to this work.
}

\begin{abstract}
Purpose: To characterize the patterns of care and potential barriers to access to care for elderly patients with oral cavity cancer in the adjuvant setting.

Methods and Materials: We performed a retrospective cohort study using the National Cancer Data Base and identified patients with resected oral cavity squamous cell carcinoma diagnosed between 2004 and 2012, who survived for $\geq 3$ months after surgery. We used logistic regression models to assess the association between age $(<70,70-79$, and $\geq 80$ years) and the receipt of adjuvant therapy within 3 months of surgery. We additionally assessed the association between patient and tumor characteristics and the receipt of adjuvant therapy among those aged $\geq 70$ years.
\end{abstract}

Results: A total of 25,829 patients were included in the study. Compared with those aged $<70$ years, older patients were more likely to have no neck dissection or have fewer lymph nodes dissected and were less likely to receive adjuvant therapy than younger patients. Among our cohort, 11,361 patients (44\%) had pathologic T3-T4 disease or N2-N3 disease, and 4185 patients (16\%) had extracapsular nodal extension or positive surgical margins. In multivariate analyses controlling for comorbidity and demographic characteristics, older age was independently associated with lower odds of receiving adjuvant radiation therapy in the subgroup with T3 or T4 disease or $\mathrm{N} 2$ or $\mathrm{N} 3$ disease and adjuvant chemoradiation therapy in the positive extracapsular nodal extension or positive surgical margin subgroup. Among elderly patients, both greater patient distance from reporting facility and older age were associated with lower odds of receiving both adjuvant radiation therapy (odds ratio 0.66 ; $95 \%$ confidence interval, $0.55-0.81$ ) and chemoradiation therapy (odds ratio $0.56 ; 95 \%$ confidence interval, $0.40-0.79$ ).

Conclusions: In a national hospital-based cohort of patients with oral cavity cancer, elderly patients were less likely to receive adjuvant radiation or chemoradiation therapy. Greater patient

Reprint requests to: C. Jillian Tsai, MD, PhD, Department of Radiation Oncology, Memorial Sloan Kettering Cancer Center, 1275 York Ave, New York, NY 10065. Tel: (631)623-4268, tsaic@ mskcc.org.

Conflict of interest: none.

Supplementary material for this article can be found at www.redjournal.org. 
distance from reporting facility, in addition to older age, was associated with lower odds of receiving both adjuvant radiation therapy and adjuvant chemoradiation therapy.

\section{Summary}

We compared patterns of adjuvant therapy in different patient age groups $(<70,70-79$, and $\ 80$ years) with resected oral cavity squamous cell carcinoma using the National Cancer Data Base. Older patients were less likely to receive adjuvant therapy, even among those with pathologic T3T4 disease or N2-N3 disease, extracapsular nodal extension, or positive margins. Both greater distance from reporting facility and older age were associated with lower odds of receiving adjuvant therapy.

\section{Introduction}

It has been estimated that 62,000 new cases of head and neck cancer, including oral cavity, pharyngeal, and laryngeal cancer, would be diagnosed in the United States in 2016 (1). Consistent with other cancer subtypes, head and neck cancer disproportionately affects elderly individuals, with a median age of diagnosis of 62 years (2). Despite this, the appropriate management of head and neck cancer in elderly patients remains relatively controversial. Older individuals often have an increased number of comorbidities, poor performance status, and limited social support, which may influence a physician's decision regarding aggressiveness of therapy because of concerns over treatment toxicity (3). Furthermore, elderly patients were often underrepresented in large randomized clinical studies that have established the standard of care in head and neck cancer (4-6). As such, generalizability of these results may be limited.

Nevertheless, a growing body of evidence suggests that a subset of elderly patients may benefit from aggressive therapy, similar to their younger counterparts. A large meta-analysis of chemotherapy in nonmetastatic head and neck cancer found that concurrent chemotherapy with radiation therapy (CRT) significantly improved survival among all comers, but a subset analysis did not find a statistically significant benefit to chemotherapy for patients aged $>70$ years (7). Contrary to these findings, a recently published cohort study using the National Cancer Data Base (NCDB) revealed that elderly patients might still benefit from concurrent chemotherapy when treated with radiation therapy (RT) in the definitive setting (8). A propensity score-matched analysis, controlling for patient and tumor characteristics, demonstrated a 5-year absolute survival difference of $15 \%$ in favor of CRT compared with RT alone among patients aged $>70$ years. This suggests that age alone should perhaps not be used to exclude patients from aggressive treatment.

Of the major head and neck subsites, oral cavity cancer is unique in that the vast majority of patients undergo upfront surgery for definitive management. As such, patients with locally advanced disease often require dual-modality treatment with adjuvant RT or triple-modality treatment with adjuvant CRT. Combined analysis of the European Organisation for Research and Treatment of Cancer (EORTC)22931 and Radiation Therapy Oncology Group (RTOG) 95-01 trials found that extracapsular nodal extension (ECE) and positive surgical margins were associated with survival benefit from CRT after surgery (9). However, the patterns of care in the adjuvant setting for elderly patients (age $\geq 70$ years) with head and neck cancer 
remain poorly described. In this study, using a large national cohort, we sought to elucidate these patterns as well as barriers to adjuvant treatment of oral cavity squamous cell carcinoma among elderly patients and to compare them with their younger counterparts.

\section{Methods and Materials}

\section{Database and cohort selection}

The NCDB is a joint program of the Commission on Cancer (CoC) of the American College of Surgeons and the American Cancer Society. It is a hospital-based registry that collects data from $>1500 \mathrm{CoC}$-accredited cancer hospitals and contains data on $70 \%$ of all incident cancer cases in the United States. The NCDB contains detailed information including demographic characteristics, disease stage, co-morbidity, radiation, surgery, and chemotherapy delivered during the first course of treatment. The data used in the study were derived from a deidentified NCDB file. The American College of Surgeons and the CoC have not verified and are not responsible for the analytical or statistical methodology used or for the conclusions drawn from these data by the investigators. The following NCDB analysis was performed with the approval of our institutional review board.

We initially queried patients with oral cavity cancers diagnosed between 2004 and 2012. We included only patients with squamous cell carcinoma using International Classification of Diseases for Oncology histology codes 8070-8076. We excluded patients who did not have known follow-up and patients who had metastatic disease. We included only patients who underwent definitive surgery and excluded patients whose timing of surgery after diagnosis was unknown. We also excluded patients who had in situ disease, who had incomplete pathologic staging, and who had received either neoadjuvant RTor chemotherapy prior to surgery. Finally, the resulting cohort was limited to patients who survived or had follow-up for $\geq 3$ months after surgery because we required RT or chemotherapy to have been delivered during this period to count as adjuvant therapy. Patients who died within this period would not have been eligible to receive adjuvant therapy.

\section{Covariates}

We included relevant patient, tumor, and treatment characteristics from the database in our analysis. Age was categorized as $<70,70$ to 79 , or $\geq 80$ years. Race was categorized as white, black, or other. Patient comorbidities were categorized as 0,1 , or 2 according to the Charlson-Deyo comorbidity score (10). Distance from facility was based on the "great circle" distance in miles between the patient's residence and the hospital that reported the case and was dichotomized into categories of $\mathbf{5 0}$ miles and $>50$ miles. Residence (metropolitan, urban, or rural) was coded according to published files by the US Department of Agriculture Economic Research Service. Median house-hold income for each patient's ZIP code of residence was derived from year 2000 US Census data. Pathologic T and N categories were based on the edition of the American Joint Committee on Cancer staging guidelines corresponding to the patient's year of diagnosis (11). Margins were considered positive if there was residual tumor, microscopic residual tumor, or macroscopic residual tumor at the surgical margins after resection of the primary tumor. We considered patients who were node negative or node positive without ECE as "ECE absent" and those who were 
node positive with ECE as "ECE present." We categorized neck dissections based on the number of nodes dissected ( $<18$ vs $\geq 18$ ) as studies have shown that this lymph node count can potentially serve as a quality metric for neck dissection (12). We considered those patients who had 0 or 1 lymph nodes dissected as not having undergone neck dissection because these patients most likely had lymph node biopsy. Finally, if a patient received RT within 3 months of definitive surgery, we considered the patient as having undergone adjuvant RT. If a patient received any systemic therapy within 3 months of definitive surgery, we considered the patient as having received adjuvant chemotherapy or cetuximab (13). Cetuximab was coded as chemotherapy during the study period. If a patient received both RT and chemotherapy during the 3-month period after definitive surgery, we considered the patient as having received adjuvant CRT.

\section{Statistical analysis}

Pearson $\chi^{2}$ tests were used to assess associations between baseline characteristics and age groups. We used logistic regression models to evaluate the association between age groups and the receipt of adjuvant therapy, as well as the association of additional patient and tumor characteristics and the receipt of adjuvant therapy among patients aged $\geq 70$ years. We included in our adjusted models relevant covariates that were selected a priori based on clinical judgment. Statistical analyses were performed using SAS Enterprise Guide (version 7.12; SAS Institute, Cary, NC).

\section{Results}

\section{Patient, tumor, and treatment characteristics}

A total of 25,829 patients were included in the study. Table E1 (available online at www.redjournal.org) shows the details of our cohort selection. Of these, 18,006 were aged $<70$ years, 5064 were aged 70 to 79 years, and 2759 were aged $\geq 80$ years. Tables $1-3$ show baseline patient, tumor, and treatment characteristics by age group. In general, older patients were more likely to be women, to be white, to have higher comorbidity scores, and to live in areas with higher median incomes. Younger patients tended to have more oral tongue cancers, whereas older patients had more gum cancers. Older patients tended to have a more advanced pathologic $\mathrm{T}$ category but more pathologic node-negative disease; however, they were also more likely to have no neck dissection or to have fewer lymph nodes $(<18)$ dissected. Older patients were less likely to receive adjuvant RTor CRT than younger patients. Figure 1 shows trends over time in receipt of adjuvant therapy by age group.

Patients who had more extensive nodal dissections ( $\geq 18$ lymph nodes dissected) were more likely to receive adjuvant RT and chemotherapy. Forty-eight percent of those with $\geq 18$ lymph nodes dissected received adjuvant RT compared with $34 \%$ of those with $<18$ lymph nodes dissected and $19 \%$ of those who did not undergo neck dissection $(P<.0001)$. Similarly, $21 \%$ of those with $\geq 18$ lymph nodes dissected received adjuvant chemoradiation therapy compared with $12 \%$ of those with <18 lymph nodes dissected and $5 \%$ of those who did not undergo neck dissection $(P<.0001)$. 


\section{Analysis of subgroups in which adjuvant therapy is warranted}

Of our cohort, 11,361 patients (44\%) had pathologic T3-T4 disease or N2-N3 disease. Of these patients, 6917 (61\%) underwent adjuvant RT within 3 months of surgery. Older patients were less likely to undergo RT: 5171 of 7894 patients (66\%) aged <70 years, 1235 of 2239 patients $(55 \%)$ aged between 70 and 79 years, and 511 of 1228 patients $(42 \%)$ aged $\geq 80$ years underwent adjuvant RT. After we adjusted for sex, race, year of diagnosis, comorbidity score, region of the country, facility type, oral cavity site, distance from facility, income, and residence type (urban, metropolitan, or rural), older patients were still less likely to undergo adjuvant RT (Table 4).

We similarly looked at the subgroup with ECE or positive surgical margins $(n=4185)$ and found that only 1495 of these patients (36\%) received adjuvant CRT within 3 months of surgery. Older patients were also less likely to undergo CRT: 1249 of 2969 patients (42\%) aged $<70$ years, 201 of 809 patients (25\%) aged between 70 and 79 years, and 45 of 407 patients (11\%) aged $\geq 80$ years underwent adjuvant CRT. Older age was independently associated with lower odds of receiving adjuvant CRT (Table 4).

\section{Sensitivity analysis excluding patients who died within 1 year of surgery}

A potential reason for omission of adjuvant therapy could be poor performance status and/or short life expectancy. Although we controlled for comorbidity score, we wanted to further evaluate whether older patients were less likely to receive adjuvant therapy even among those who survived $\geq 1$ year after surgery.

In the subgroup with pathologic T3-T4 disease or N2-N3 disease, 8252 patients survived $\geq 1$ year after surgery. Among these patients, those aged 70 to 79 years had lower odds (odds ratio $0.64 ; 95 \%$ confidence interval $[\mathrm{CI}], 0.57-0.73 ; P<.0001$ ) of receiving adjuvant RT than those aged $<70$ years, after adjustment for relevant covariates. Those aged $\geq 80$ years had even lower odds (odds ratio $0.4 ; 95 \% \mathrm{CI}, 0.34-0.48 ; P<.0001$ ) of receiving adjuvant RT than those aged $<70$ years.

Similarly, among patients with ECE or positive surgical margins who lived $\geq 1$ year after surgery ( $\mathrm{n}=2883$ ), those aged 70 to 79 years had lower adjusted odds (odds ratio $0.54 ; 95 \%$ CI, $0.43-0.69 ; P<.0001)$ of receiving adjuvant CRT than those aged $<70$ years, and those aged $\geq 80$ years had even lower odds (odds ratio $0.18 ; 95 \%$ CI, $0.11-0.29 ; P<.0001$ ) of receiving adjuvant $\mathrm{CRT}$ than those aged $<70$ years.

\section{Factors associated with receipt of adjuvant therapy among patients aged $>70$ years}

Among older patients (age $\geq 70$ years), we determined factors associated with receipt of adjuvant RT in those with pathologic T3-T4 disease or N2-N3 disease and receipt of adjuvant CRT in those with ECE or positive surgical margins. Both greater patient distance from reporting facility and older age were associated with lower odds of receiving both adjuvant RT and adjuvant CRT (Table 5). In addition, patients who had lower comorbidity scores and who were treated in community cancer programs and in the Northeast were more likely to receive adjuvant RT. Patients who were treated at an academic institution were more likely to live $>50$ miles away from the reporting facility than patients treated in 
community, comprehensive community, and integrated network cancer programs. Among patients aged $\geq 70$ years with pathologic T3-T4 disease or N2-N3 disease, 33\% of those treated at an academic facility lived $>50$ miles away compared with $11 \%$ of those treated at a nonacademic facility $(P<.0001)$. Similarly, among patients aged $\geq 70$ years with ECE or positive surgical margins, $33 \%$ of those treated at an academic facility lived $>50$ miles away compared with $8 \%$ of those treated at a nonacademic facility.

\section{Discussion}

In this study we characterized the clinical characteristics, treatment practices, and potential barriers to access of care for oral cavity cancer in the adjuvant setting among patients of different age groups. Our cohort was generated using the NCDB, which affords us a large, nationally representative sample of patients. Among the entire cohort, we found that elderly patients were significantly less likely to receive adjuvant treatment. Even in the subset of patients with pathologic T3-T4 disease or N2-N3 disease and on multi-variate analysis controlling for comorbidity score and other demographic characteristics, elderly patients were less likely to receive adjuvant RT. Furthermore, we examined the relationship between age and treatment among patients with clinical indications for adjuvant CRT as established by EORTC 22931 and RTOG 95-01 $(5,6,9)$ and found that elderly patients were also less likely to receive CRT than their younger counterparts.

A primary concern regarding aggressive treatment in elderly patients is increased morbidity due to treatment toxicities $(14,15)$. However, several studies have suggested that surgery followed by adjuvant RT or CRT in the definitive setting is well tolerated among elderly patients with head and neck cancer if they are carefully selected with respect to comorbidity status (16-19). Huang et al (19) published the largest modern single-institution cohort study examining patterns of care in elderly patients with head and neck cancer. They found that elderly patients were more likely to be women, to have earlier-stage disease, and to present with N0 nodal status, comparable to our findings in the NCDB cohort. Furthermore, they found that a smaller proportion of these patients were treated with curative intent compared with younger patients. Despite this, elderly patients had no increased rate of RT treatment interruptions, treatment-related deaths, or late toxicities. However, their study was limited by lack of comorbidity and performance status data.

Pignon et al (20) previously evaluated the RT toxicity patterns with respect to age among 1300 patients enrolled on EORTC trials and found no significant difference in the rate of objective acute mucositis, weight loss, or late toxicity occurrence among age groups from 50 to 75 years. In our study we selected only patients who had undergone upfront definitive surgical resection and survived $\geq 3$ months after surgery, presumably enriching our sample for those deemed medically fit to undergo aggressive treatment despite advanced age. Furthermore, we performed a sensitivity analysis of those surviving $\geq 1$ year after treatment and found that elderly patients with longer life expectancies were still less likely to receive adjuvant RT or CRT than their younger counterparts.

We identified several demographic factors associated with receipt of adjuvant RT or CRT, even after controlling for patient age and disease characteristics. Distance from reporting 
facility was inversely associated with likelihood of receiving both RT and CRT. Previous research has identified impaired access to transportation as a barrier to cancer treatment among elderly patients (21). This finding is also consistent with the body of literature in breast cancer, which has shown that women living farther from a treatment facility are less likely to receive RT either after breast-conserving surgery (22-24) or after mastectomy (25). Although all of the facilities in the NCDB are CoC-accredited, we found that academic facilities were less likely to administer adjuvant therapy. While patients living at a greater distance from the reporting academic facility could be a reason for this observation, the difference in reimbursement and/or incentive structure between nonacademic and academic institutions might have also accounted for different patterns of adjuvant therapy use.

Contrary to distance, more recent year of diagnosis was associated with a trend toward greater likelihood of receiving adjuvant therapy, likely due in large part to changing practice patterns after publication of the results of EORTC 22931 and RTOG 95-01 in $2004(5,6)$. More contemporary increases in CRT use are perhaps reflective of presumed improvements in supportive care, more conformal radiation techniques, or less toxic systemic therapies. In a recent analysis of the linked Surveillance, Epidemiology, and End Results (SEER) Medicare database, Baxi et al (26) found that CRT use among head and neck cancer patients aged $\searrow 65$ years increased from $29 \%$ in 2001 to $61 \%$ in 2009. Furthermore, older age was associated with decreased likelihood of receiving CRT before 2006 but not afterward, which Baxi et al postulate is because of increased use of cetuximab in elderly patients. Specifically, cetuximab was the most commonly used concurrent systemic agent among elderly patients after its Food and Drug Administration approval in 2006, and its rise coincided with the decline in use of platinum agents over the same period (26). Similarly, in our NCDB cohort, the rate of adjuvant CRT use among elderly patients (aged $\geq 70$ years) with ECE or positive surgical margins increased from 13\% in the period of 2004 to 2007 to $24 \%$ in 2008 to 2012, during which cetuximab was more commonly used. However, age remained a significant predictor of treatment even after controlling for year of diagnosis. The difference in findings may be reflective of differences in cohort selection, as Baxi et al included patients who underwent RTor CRT in either the definitive or adjuvant setting. In the purely adjuvant setting, our data suggest that providers may still be less willing to prescribe RTor CRT on the basis of older age alone.

The NCDB provides a large cohort from which to investigate treatment patterns across the country and trends over time. It is uniquely equipped to investigate differential treatment patterns across age groups, as it includes both elderly and non-elderly patients, unlike the SEER-Medicare database. However, several limitations exist with any such analysis. It is subject to the inherent biases of the database's retrospective nature, including the potential for incomplete or inaccurate coding. Details regarding specific systemic drug regimens are not available. Furthermore, the receipt of RT or CRT may be influenced by factors not captured in the database, such as performance status and provider and patient preference. Similarly, detailed analysis of treatment-related adverse effects and toxicity management, including treatment breaks, is not feasible with the database but would be important to help determine tolerability of adjuvant RT and CRT. There has also been concern that registry data do not fully reflect all instances of RT delivery. An analysis of SEER data found RT underascertainment rates among patients with breast cancer on the order of $10 \%$ to $30 \%$ 
(27). However, older age was associated with improved accuracy of RT coding in this study, which would have underestimated any inverse association between age and receipt of adjuvant therapy.

Last, an outcomes analysis was outside the scope of this study. As such, we are unable to comment on the survival benefit of adjuvant RT or CRT among elderly patients. Future clinical trials specifically designed to investigate whether similar tumor characteristics warrant aggressive adjuvant treatment in elderly patients will be crucial to answer this question. Nevertheless, previous research has suggested that RT and CRT are well tolerated among carefully selected elderly patients with head and neck cancer (16-20) and that age itself should not preclude consideration of treatment. Further advances in supportive care, adaptive radiation techniques, and targeted therapy for head and neck cancer (28) may continue to increase the use of adjuvant therapy in elderly patients.

\section{Conclusion}

In a national cohort of patients with oral cavity cancer, elderly patients were significantly less likely to receive adjuvant RT or CRT after controlling for comorbidity status and demographic characteristics. Among those aged $\geq 70$ years, both greater patient distance from reporting facility and older age were associated with lower odds of receiving both adjuvant RT and adjuvant chemoradiation therapy. These results add to the growing body of evidence showing that elderly patients are disproportionately offered less aggressive treatment.

\section{Supplementary Material}

Refer to Web version on PubMed Central for supplementary material.

\section{Acknowledgments}

Supported by the KL2 Mentored Career Development Award of the Stanford Clinical and Translational Science Award to Spectrum (NIH KL2 TR 001083) (to E.L.P.) and National Institutes of Health-National Cancer Institute Cancer Center Support Grant P30 CA008748 (to C.J.T.).

\section{References}

1. Siegel RL, Miller KD, Jemal A. Cancer statistics, 2016. CA Cancer J Clin 2016;66:7-30. [PubMed: 26742998]

2. Howlader N, Noone AM, Krapcho M, et al. (eds). SEER Cancer Statistics Review, 1975-2013, National Cancer Institute Bethesda, MD Available at: http://seer.cancer.gov/csr/1975_2013/, based on November 2015 SEER data submission, posted to the SEER web site, April 2016. Accessed March 21, 2017.

3. Chen RC, Royce TJ, Extermann M, et al. Impact of age and comorbidity on treatment and outcomes in elderly cancer patients. Semin Radiat Oncol 2012;22:265-271. [PubMed: 22985808]

4. Siddiqui F, Gwede CK. Head and neck cancer in the elderly population. Semin Radiat Oncol 2012;22:321-333. [PubMed: 22985815]

5. Bernier J, Domenge C, Ozsahin M, et al. Postoperative irradiation with or without concomitant chemotherapy for locally advanced head and neck cancer. N Engl J Med 2004;350:1945-1952. [PubMed: 15128894] 
6. Cooper JS, Pajak TF, Forastiere AA, et al. Postoperative concurrent radiotherapy and chemotherapy for high-risk squamous-cell carcinoma of the head and neck. N Engl J Med 2004;350:1937-1944. [PubMed: 15128893]

7. Pignon JP, le Maître A, Maillard E, et al. Meta-analysis of chemo-therapy in head and neck cancer (MACH-NC): An update on 93 randomised trials and 17,346 patients. Radiother Oncol 2009;92:414. [PubMed: 19446902]

8. Amini A, Jones BL, McDermott JD, et al. Survival outcomes with concurrent chemoradiation for elderly patients with locally advanced head and neck cancer according to the National Cancer Data Base. Cancer 2016;122:1533-1543. [PubMed: 26969811]

9. Bernier J, Cooper JS, Pajak TF, et al. Defining risk levels in locally advanced head and neck cancers: A comparative analysis of concurrent postoperative radiation plus chemotherapy trials of the EORTC (\#22931) and RTOG (\# 9501). Head Neck 2005;27:843-850. [PubMed: 16161069]

10. Deyo RA, Cherkin DC, Ciol MA. Adapting a clinical comorbidity index for use with ICD-9-CM administrative databases. J Clin Epidemiol 1992;45:613-619. [PubMed: 1607900]

11. Edge SB, Compton CC. The American Joint Committee on Cancer: The 7th edition of the AJCC cancer staging manual and the future of TNM. Ann Surg Oncol 2010;17:1471-1474. [PubMed: 20180029]

12. Divi V, Chen MM, Nussenbaum B, et al. Lymph node count from neck dissection predicts mortality in head and neck cancer. J Clin Oncol 2016;34:3892-3898. [PubMed: 27480149]

13. National Cancer Data Base. Data Dictionary PUF 2014 Available at: http://ncdbpuf.facs.org/node/ 259?q=print-pdf-all.

14. Baumann M Is curative radiation therapy in elderly patients limited by increased normal tissue toxicity? Radiother Oncol 1998;46: 225-227. [PubMed: 9572614]

15. Syrigos KN, Karachalios D, Karapanagiotou EM, et al. Head and neck cancer in the elderly: An overview on the treatment modalities. Cancer Treat Rev 2009;35:237-245. [PubMed: 19100689]

16. Huguenin P, Sauer M, Glanzmann C, et al. Radiotherapy for carcinomas of the head and neck in elderly patients. Strahlenther Onkol 1996;172:485-488. [PubMed: 8830810]

17. Airoldi M, Cortesina G, Giordano C, et al. Postoperative adjuvant chemoradiotherapy in older patients with head and neck cancer. Arch Otolaryngol Head Neck Surg 2004;130:161-166. [PubMed: 14967744]

18. Kruse AL, Bredell M, Luebbers HT, et al. Head and neck cancer in the elderly: A retrospective study over 10 years (1999-2008). Head Neck Oncol 2010;2:25. [PubMed: 20923547]

19. Huang SH, O’Sullivan B, Waldron J, et al. Patterns of care in elderly head-and-neck cancer radiation oncology patients: A single-center cohort study. Int J Radiat Oncol Biol Phys 2011;79:46-51. [PubMed: 20395066]

20. Pignon T, Horiot JC, Van den Bogaert W, et al. No age limit for radical radiotherapy in head and neck tumours. Eur J Cancer 1996;32A: 2075-2081. [PubMed: 9014748]

21. Goodwin JS, Hunt WC, Samet JM. Determinants of cancer therapy in elderly patients. Cancer 1993;72:594-601. [PubMed: 8319193]

22. Athas WF, Adams-Cameron M, Hunt WC, et al. Travel distance to radiation therapy and receipt of radiotherapy following breast-conserving surgery. J Natl Cancer Inst 2000;92:269-271. Available at: http://ncdbpuf.facs.org/node/259?qZprint-pdf-all. Accessed March 21, 2017. [PubMed: 10655446]

23. Nattinger AB, Kneusel RT, Hoffmann RG, et al. Relationship of distance from a radiotherapy facility and initial breast cancer treatment. J Natl Cancer Inst 2001;93:1344-1346. [PubMed: 11535710]

24. Voti L, Richardson LC, Reis IM, et al. Treatment of local breast carcinoma in Florida: The role of the distance to radiation therapy facilities. Cancer 2006;106:201-207. [PubMed: 16311987]

25. Punglia RS, Weeks JC, Neville BA, et al. Effect of distance to radiation treatment facility on use of radiation therapy after mastectomy in elderly women. Int J Radiat Oncol Biol Phys 2006;66: 5663. [PubMed: 16814955]

26. Baxi SS, O'Neill C, Sherman EJ, et al. Trends in chemoradiation use in elderly patients with head and neck cancer: Changing treatment patterns with cetuximab. Head Neck 2016;38:E165-E171.

[PubMed: 25535104] 
27. Jagsi R, Abrahamse P, Hawley ST, et al. Underascertainment of radiotherapy receipt in Surveillance, Epidemiology, and End Results registry data. Cancer 2012;118:333-341. [PubMed: 21717446]

28. Seiwert TY, Burtness B, Mehra R, et al. Safety and clinical activity of pembrolizumab for treatment of recurrent or metastatic squamous cell carcinoma of the head and neck (KEYNOTE-012): An open-label, multicentre, phase 1b trial. Lancet Oncol 2016;17:956-965. [PubMed: 27247226] 


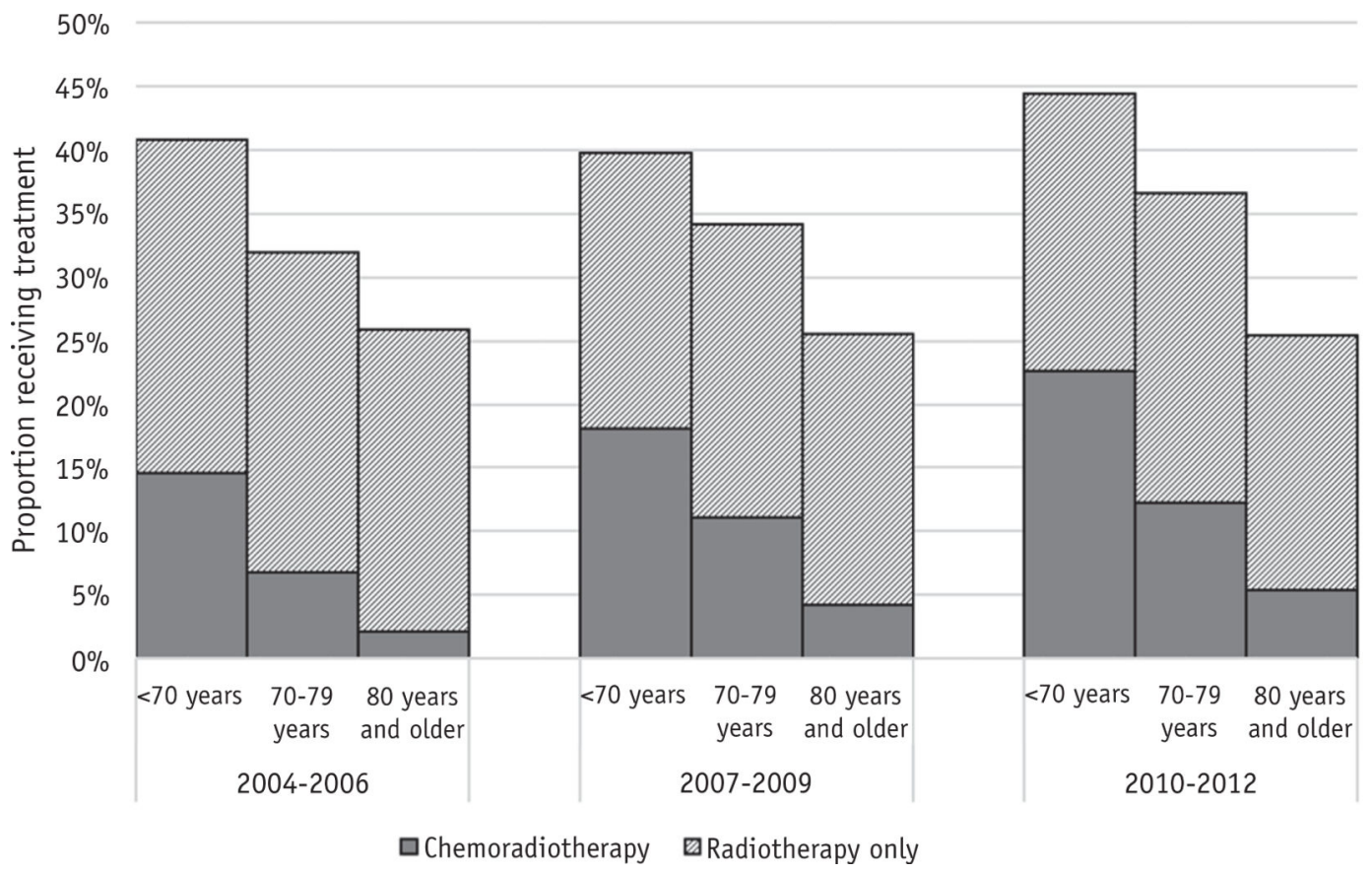

Fig. 1.

Trends in receipt of adjuvant radiation and chemoradiation therapy by age group among patients with resected oral cavity squamous cell cancer from the National Cancer Data Base. 


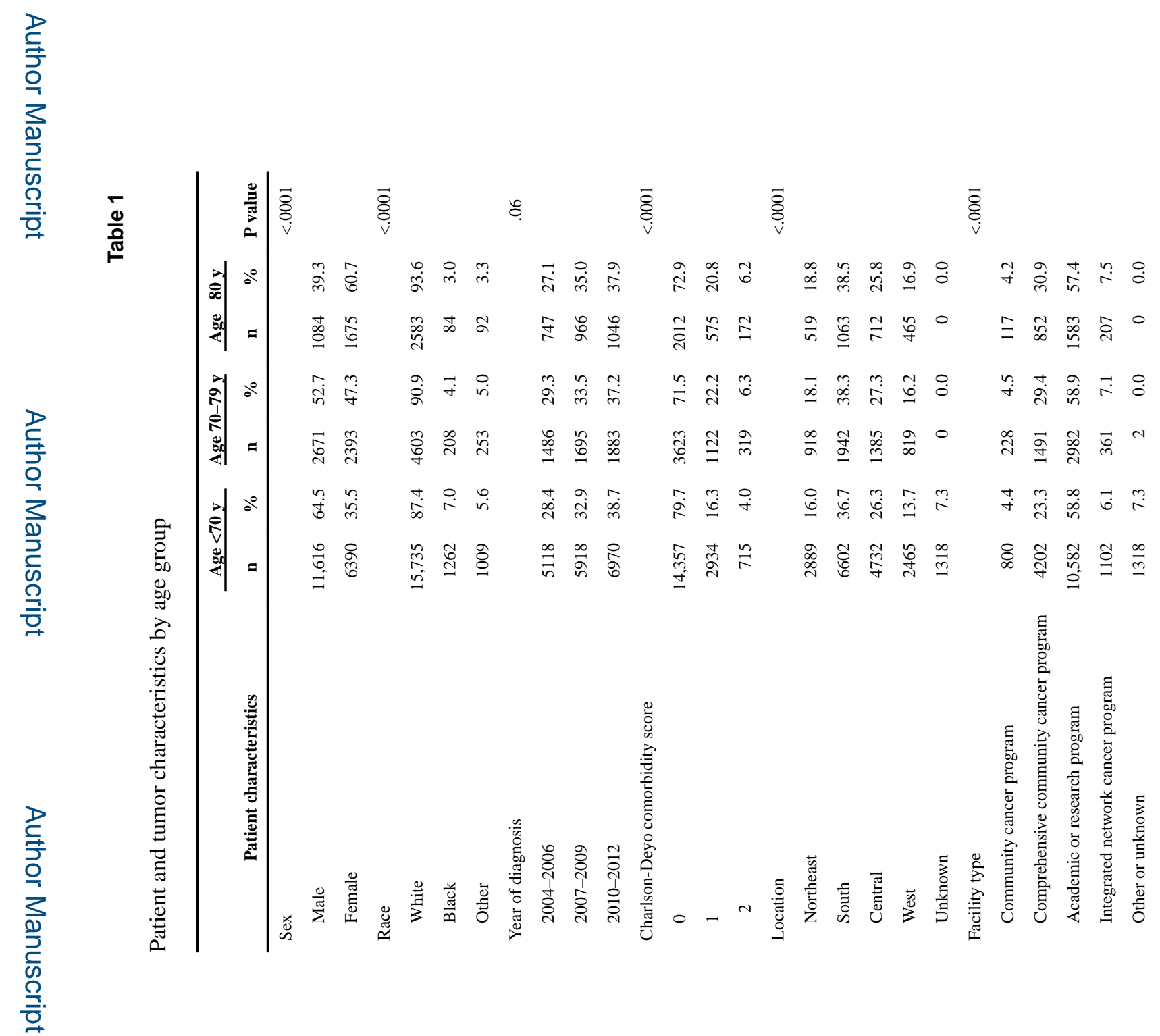

Int J Radiat Oncol Biol Phys. Author manuscript; available in PMC 2018 November 17. 

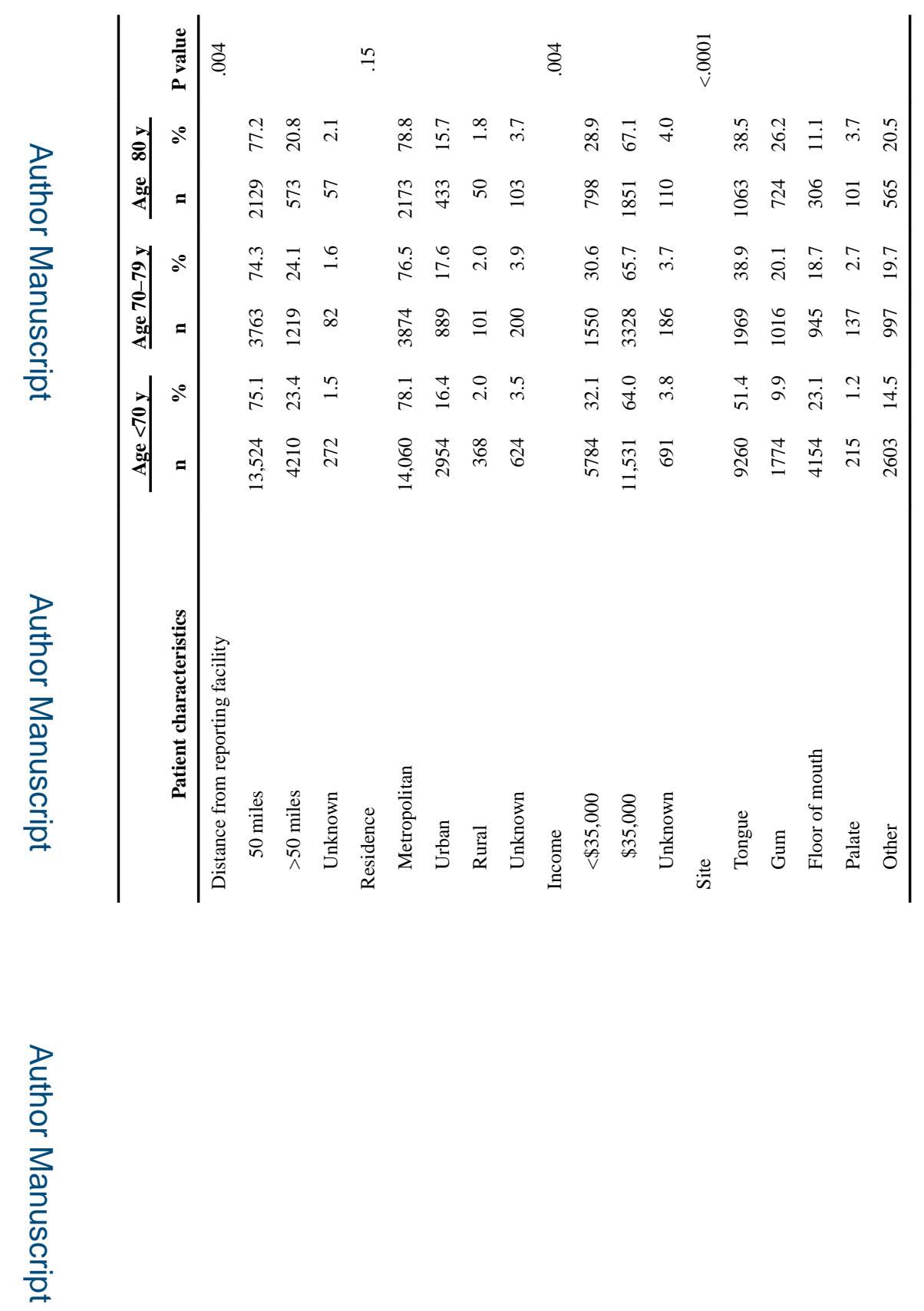

로을

Int J Radiat Oncol Biol Phys. Author manuscript; available in PMC 2018 November 17. 


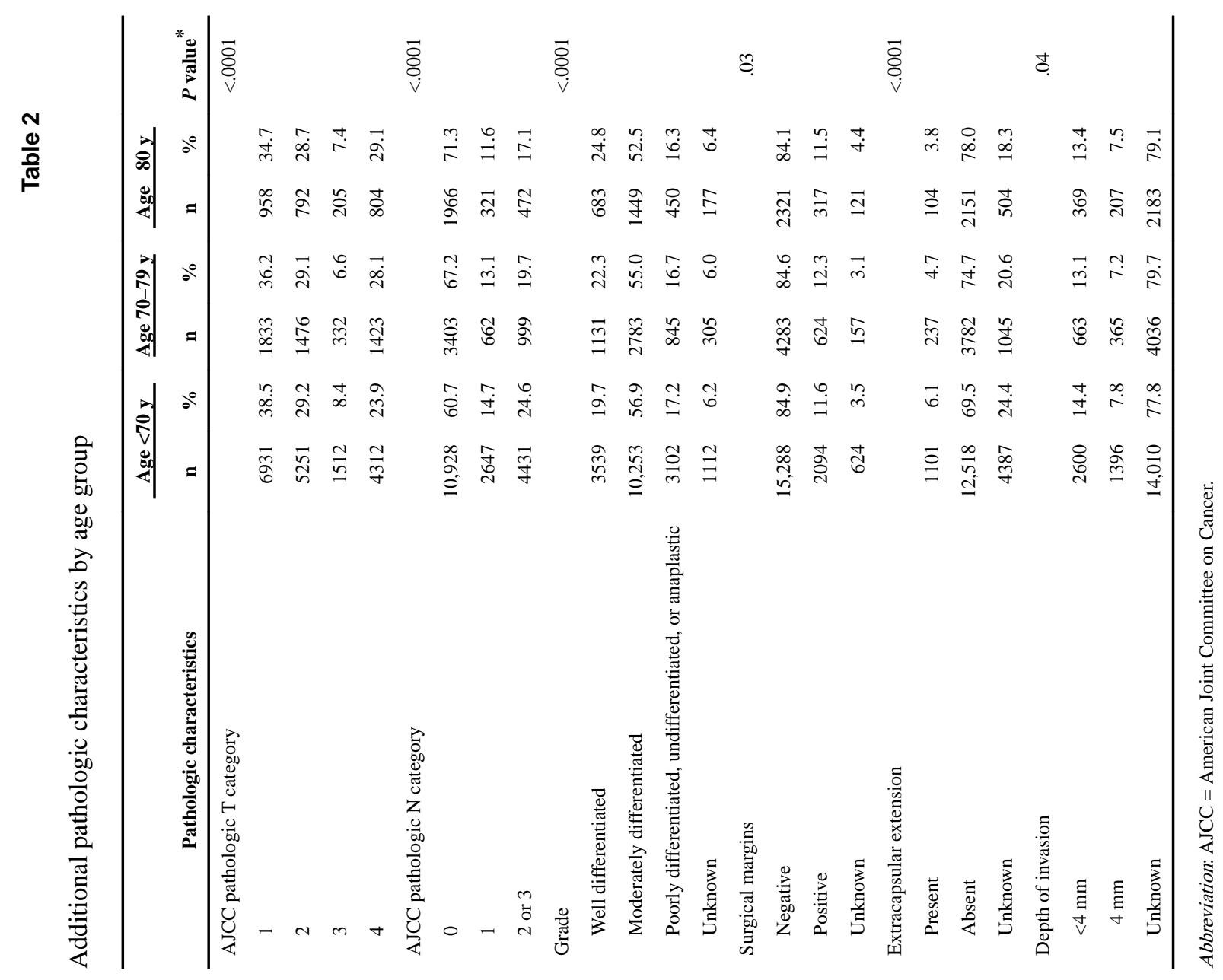

Int J Radiat Oncol Biol Phys. Author manuscript; available in PMC 2018 November 17. 


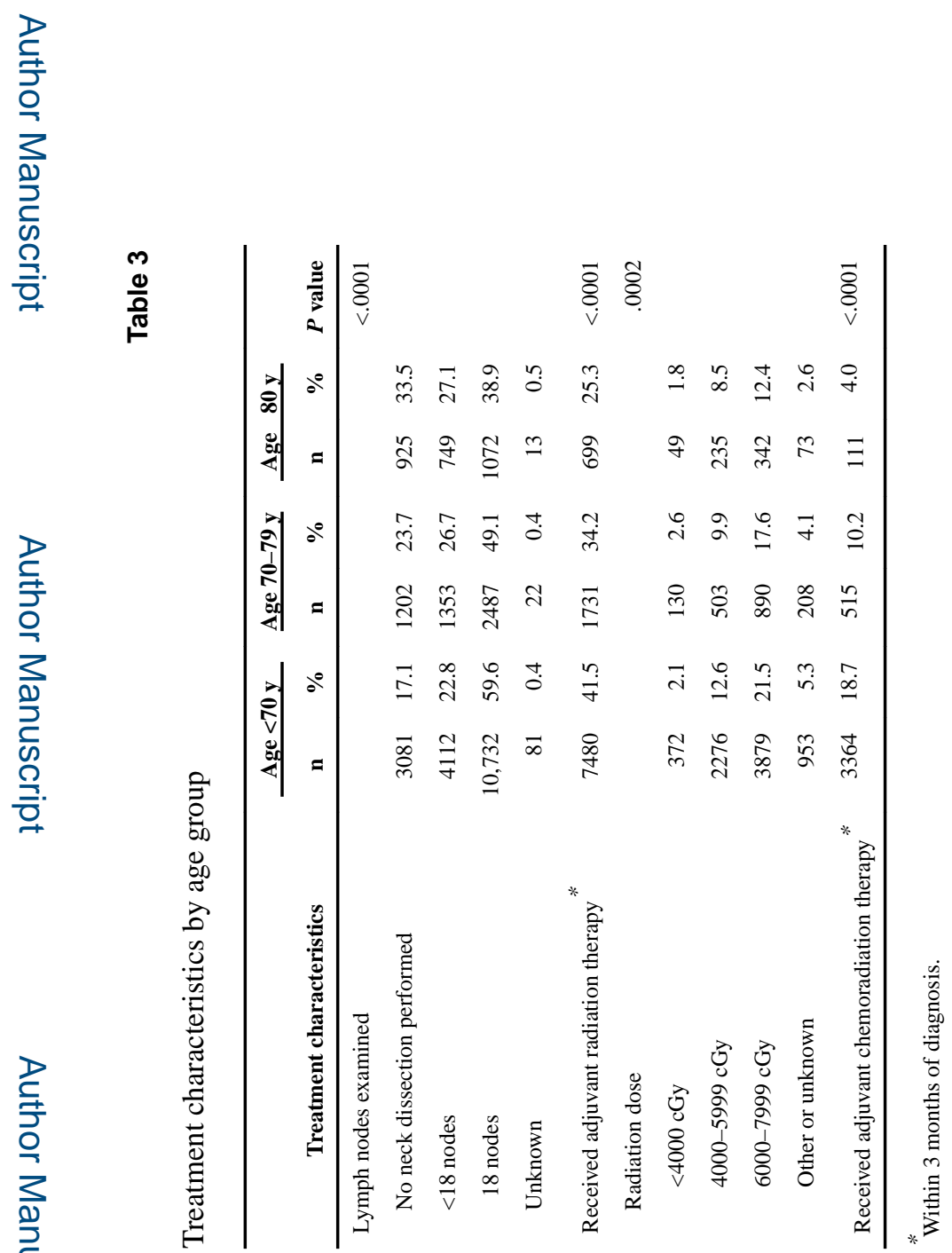




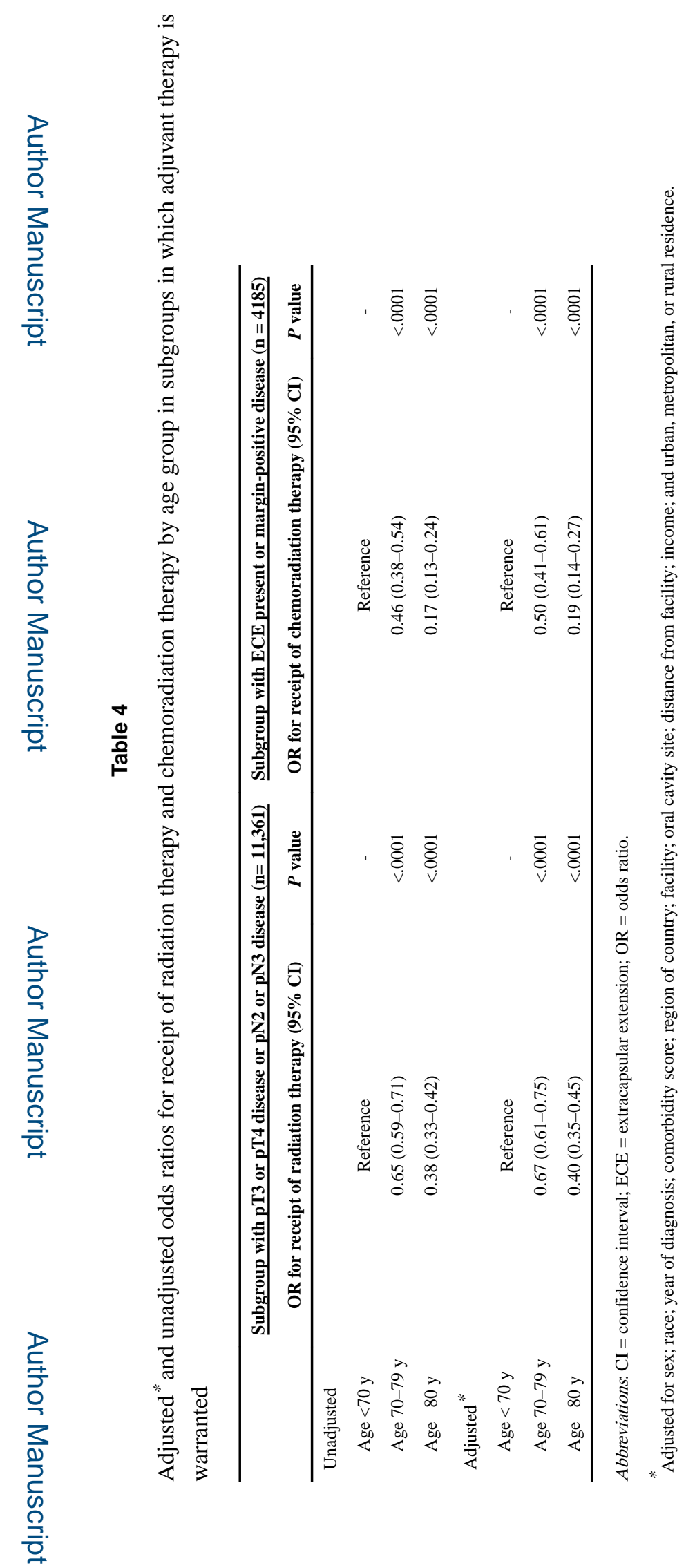

Int J Radiat Oncol Biol Phys. Author manuscript; available in PMC 2018 November 17. 


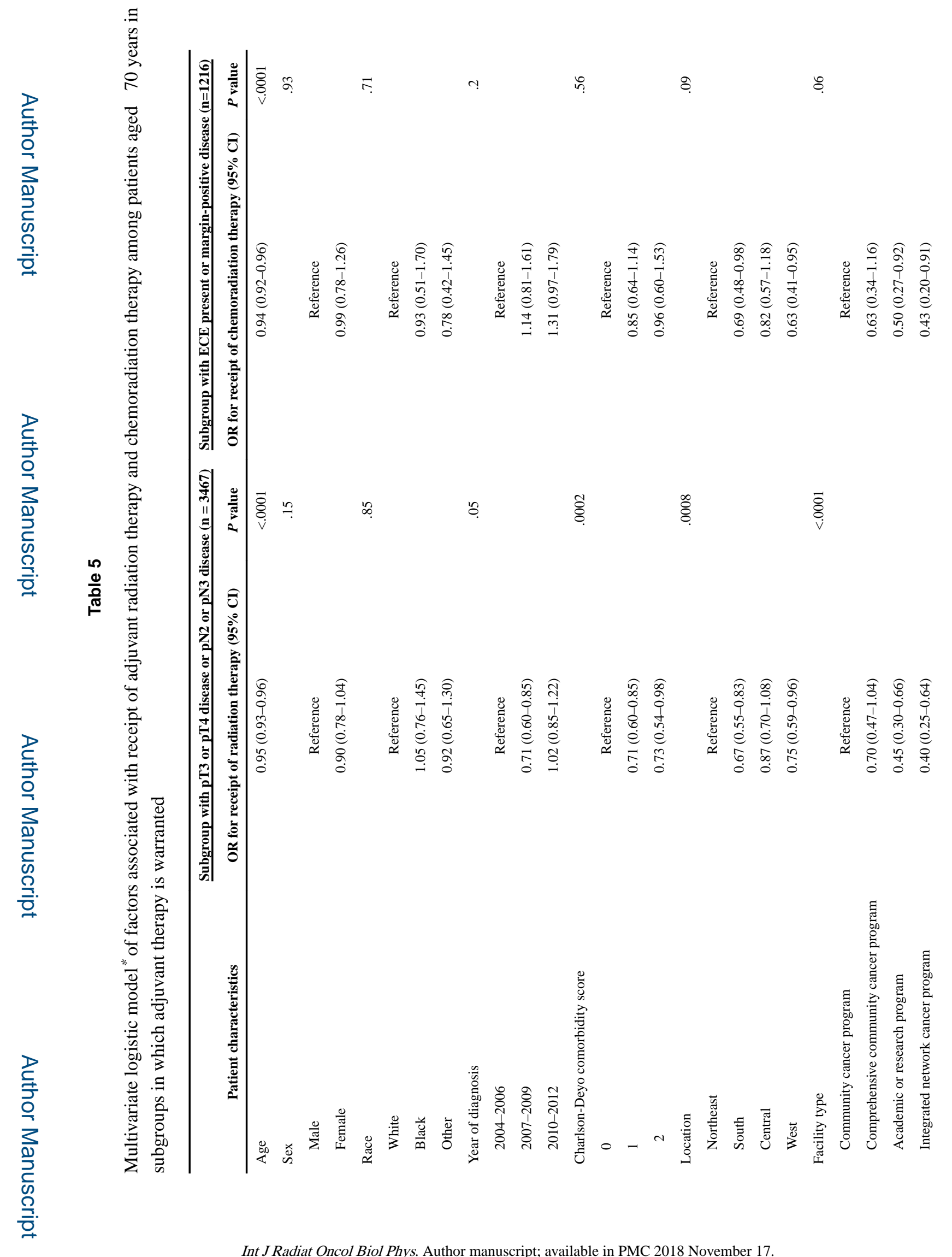


Pollom et al.

Page 19

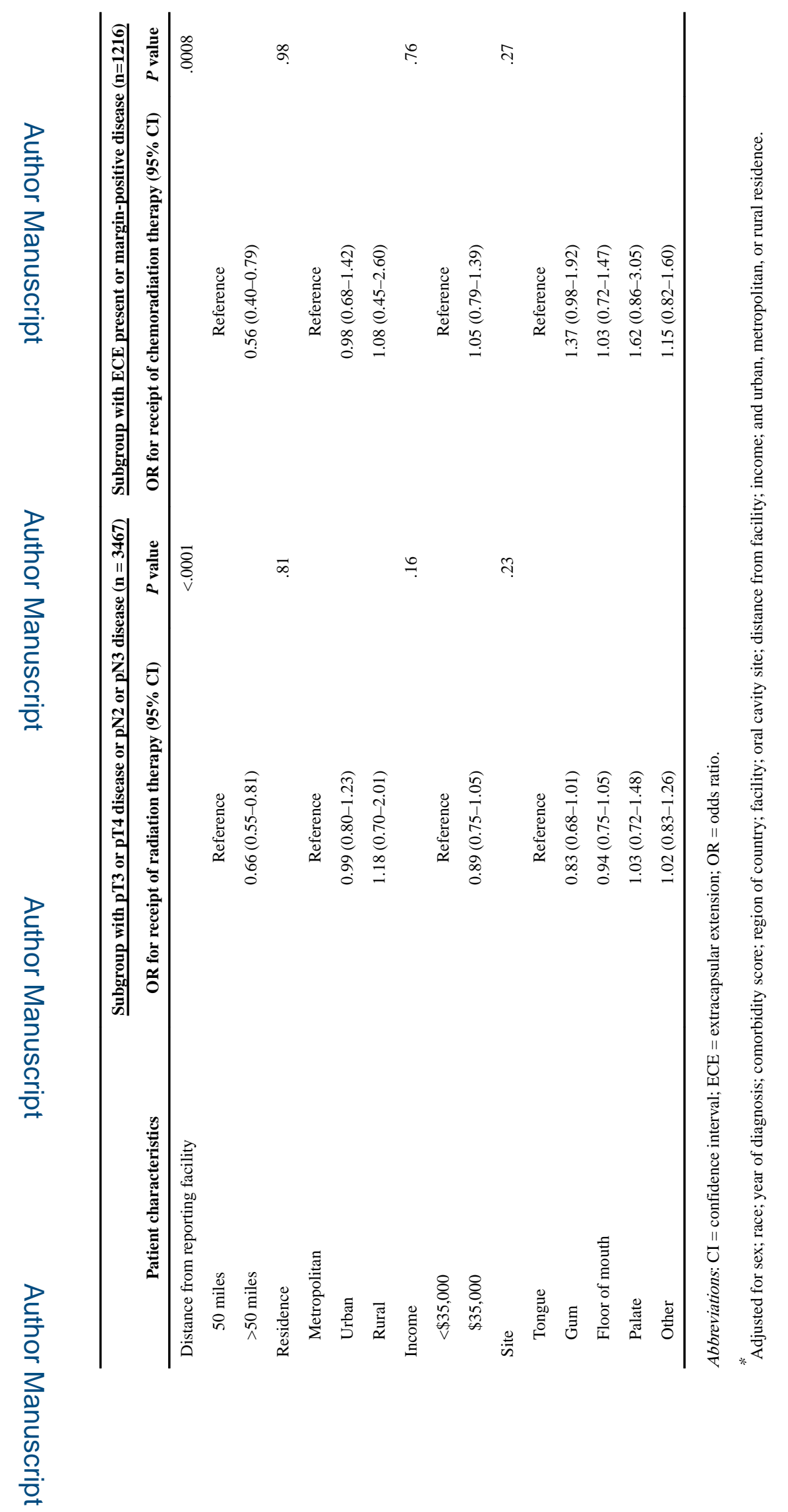

Int J Radiat Oncol Biol Phys. Author manuscript; available in PMC 2018 November 17. 